\title{
TRS: a program to calculate Landau levels and direct dipole transitions in uniaxially stressed semiconductors
}

\author{
Joachim Schmitz ${ }^{1}$, Hans-Rainer Trebin ${ }^{2}$ \\ Institut für Theoretische und Angewandte Physik, Universität Stuttgart, Pfaffenualdring 57, W-7000 Sturtgart 80. (ierman!
}

and

Ulrich Rössler ${ }^{3}$

Institut für Theoretische Physik, Universität Regenshurg, Universitätsstrasse 31, W-8400 Regensburg. German!

Received 16 February 1989

\begin{abstract}
Quantum resonances in the valence bands of semiconductors under uniaxial stress provide very detailed information on the band parameters if the experimental data can be analyzed on the basis of an adequate theoretical model. Trebin and Rössler developed such a model for narrow-gap semiconductors with a zincblende lattice and applied it to InSb. Using their theoretical results they wrote a program for the numerical evaluation of energy eigenvalues, wave functions and osciliator strengths for direct inter- and intraband dipole transitions. It is based on an effective Hamiltonian constructed by invariant expansion and describes the Landau levels in an eightfold space of valence and conduction bands when uniaxial stress and magnetic field are applied along the [001] direction. All eigenstates are evaluated for any three values of wave vector, magnetic field and stress. Recently, Schmitz made several improvements and additions to this program including a complete user interface making the program easy to use, adaptable to a wide variety of computers, and allowing calculations done systematically.
\end{abstract}

\section{PROGRAM SUMMARY}

Title of program: TRS

Catalogue number: $\mathrm{ACBH}$

Program obtainable from: CPC Program Library, Queen's University of Belfast, N. Ireland (see application form in this issue), or Joachim Schmitz, Institut für Theoretische und Angewandte Physik, Universität Stuttgart. Pfaffenwaldring 57. W-7000 Stuttgart 80, Germany,joa@ds0ita51.bitnet

Licensing provisions: none

joa@ds0ita51.bitnet

trebin@ds0ita51.bitnet

roessler@vax1.rz.uni-regensburg.dbp.de
Computer for which the program is designed and others in which it has been tested:

Computers: (1) VAX II/GPX (original design); (2) Comparex 8/89 (later made operable): (3) Atari 1040st (later made operable)

Installations: (1,3) Institut für Theoretische und Angewandte Physik, Universität Stuttgart, Pfaffenwaldring 57, W-7000 Stuttgart 80, Germany; (2) Regionales Rechenzentrum der Universität Stuttgart. Allmandring 30, W-7000 Stuttgart 80 , Germany

Operating systems under which the program is executed: (1) VAX/VMS 4.6; (2) VM/CMSL 4.421; (3) TOS 
Programming language used: standard FORTRAN 77

Memory required to execute with typical data:(1) 434 Kbytes; (2) 1.4 Mbytes; (3) 370 Kbytes (sizes of major arrays reduced to half)

No. of bits in a word: $(1,2) 32$; (3) 16

No. of processors used: 1

Has the code been vectorised? no

Peripherals used: terminal, disk

No. of lines in distributed program, including test deck, etc.: 5193 (computer (1))

Separate documentation available: TRS User's and Programmer's Guide; no. of pages: 76; available from: CPC Program Library, or Joachim Schmitz, Institut für Theoretische und Angewandte Physik, Universität Stuttgart, Pfaffenwaldring 57, W-7000 Stuttgart 80, Germany,joa@ds0ita51.bitnet

Keywords: narrow-gap semiconductors, zincblende lattice, Landau levels, uniaxially stress parallel to magnetic field, effective Hamiltonian, invariant expansion, eightfold space of valence band and lowest conduction band, normal and inverted bands, energy eigenvalues, eigenvectors, direct interand intraband dipole transitions, oscillator strengths, $\Gamma$-point, [001] crystal axis

\section{Nature of physical problem}

An effective Hamiltonian constructed by invariant expansion is used to calculate Landau levels and wave functions in narrow-gap semiconductors with a zincblende lattice under uniaxial stress [4]. It is based on an eightfold space of uppermost valence and lowest conduction bands at the center of the Brillouin zone and its vicinity. The wave functions are further used to calculate the oscillator strengths of direct interand intraband dipole transitions. Thus the TRS program is a valuable tool for the experimentalist to analyze quantum resonances measured in semiconductors.

\section{LONG WRITE-UP}

\section{Introduction}

Detailed information on the band parameters of a semiconductor can be obtained from quantum resonances in uniaxially stressed crystals. To this end the experimental data must be analyzed on the basis of a theoretical model. This model was provided by Trebin and Rössler [3,4] for narrow-gap semiconductors with a zincblende
Method of solution

The matrix elements of the Hamiltonian are set up one by one according to the equations derived from the theory [2,3]. Then the resulting matrix is diagonalized using the $\mathrm{QL}$ method. Energy eigenvalues and eigenvectors are further used in the calculation of oscillator strengths.

Restrictions on the complexity of the problem

The dimensions of arrays are set to include Landau levels with oscillator quantum number up to $n_{a} \leq 39$ ( $n_{a} \leq 19$ on computer (3)). Adaptions are easily made. Due to the limitations in the Kane-model [1] and the underlying perturbation theory, the program is only suitable for eigenstates in the vicinity of the $\Gamma$-point. Transitions are restricted to direct dipole transitions. All calculations are based on a geometry with magnetic field and uniaxial stress being parallel to the [001] crystal axis.

Typical running time

Execution times depend strongly on the maximum Landau oscillator quantum number and the number of transitions chosen. The test run described in the long write-up requires (1) $25.68 \mathrm{~s}$, (2) $2.06 \mathrm{~s}$, (3) $11 \mathrm{~min} 17 \mathrm{~s}$, on the respective computers.

Unusual features of the program

TRS contains a subroutine which clears the screen of the terminal before displaying a new page of text. This action is not essential to the operation of the program and may be entirely omitted. In order to preserve the intended screen display it must be adapted to the specific device used.

References

11] E.O. Kane, in: Semiconductors and Semimetals, vol. 1, eds. R.K. Willardson and A.C. Beer (Academic, New York; 1966), p. 75

[2] H.-R. Trebin, U. Rössler and R. Ranvaud, Phys. Rev, B 20 (1979) 686.

[3] H.-R. Trebin, W. Wolfstädter, H. Pascher and H. Häfele, Phys. Rev. B 37 (1988) 10249.

[4] G.L. Bir and G.E. Pikus, Symmetry and Strain-Induced Effects in Semiconductors (Wiley, New York, 1974).

lattice. It is based on an effective Hamiltonian constructed by invariant expansion. We give a concise description of the theory followed by a comprehensive review of the standard FORTRAN 77 program TRS we developed using our theoretical results. TRS is easy to use and adaptable to a wide variety of computers. Since calculations can be done systematically it is a valuable tool to analyze band structures in semiconductors. The 
program is fully explained in our separate manual "TRS User's and Programmer's Guide" on 76 pages.

\section{Physical problem}

In this section we summarize some elements of the theory concerning the effective-mass Hamiltonian in zincblende-type semiconductors. We study the bands in the vicinity of the fundamental edge which lies at the center $\Gamma$ of the Brillouin zone. To apply the Hamiltonian to materials with small energy gaps and small spin-orbit coupling we include the following Bloch states closest to the fundamental gap and consider them quasidegenerate (Kane model, see ref. [1]):

- lowest conduction band $\psi_{m}^{\mathrm{c}}, m= \pm \frac{1}{2}$, twofold degenerate, transform according to the irreducible representation $\Gamma_{6}$ of the point group $T_{d}$ (zincblende);

- uppermost valence band $\psi_{m}^{\mathrm{v}}, m= \pm \frac{3}{2}, \pm \frac{1}{2}$, fourfold degenerate, $\Gamma_{8}$ symmetry;

- split-off band $\psi_{m}^{s}, m= \pm \frac{1}{2}$, twofold degenerate, $\Gamma_{7}$ symmetry.

At $\boldsymbol{k}=\mathbf{0}$ these eight states are completely decoupled in the absence of external perturbations. An effective-mass Hamiltonian describes mixing among the states due to magnetic and strain interaction. The interactions are represented by a matrix Hamiltonian $\mathscr{H}(\mathbf{K})$ of the eight basis functions where $\mathbf{K}$ is a general tensor denoting the set of components in $\boldsymbol{k}$ (wave vector), $\boldsymbol{H}$ (magnetic field), $\epsilon$ (distortion tensor). With respect to the basis chosen above, the matrix Hamiltonian falls into nine blocks,

$\mathscr{H}=\left(\begin{array}{ccc}\mathscr{H}^{\mathrm{cc}} & \mathscr{H}^{\mathrm{cv}} & \mathscr{H}^{\mathrm{cs}} \\ \mathscr{H}^{\mathrm{vc}} & \mathscr{H}^{\mathrm{vv}} & \mathscr{H}^{\mathrm{vs}} \\ \mathscr{H}^{\mathrm{sc}} & \mathscr{H}^{\mathrm{sv}} & \mathscr{H}^{\mathrm{ss}}\end{array}\right)$,

consisting of intraband matrices $\mathscr{H}^{\mathrm{cc}}, \mathscr{H}^{\mathrm{vv}}, \mathscr{H}^{\mathrm{ss}}$ and interband matrices $\mathscr{H}^{\mathrm{cv}}, \mathscr{H}^{\mathrm{cs}}$, etc. This Hamiltonian matrix $\mathscr{H}(\mathbf{K})$ is solely constructed by symmetry considerations. According to the theory of invariants (see ref. [5]) $\mathscr{H}^{\alpha \beta}(\mathbf{K})$ can be ex- panded into products of $l_{\alpha \alpha} \times l_{\beta}$ matrices $X_{l}^{(\kappa)}$ and irreducible components of $\mathbf{K}_{l}^{(\kappa)^{*}}$.

$\mathscr{H}^{\alpha \beta}(\mathbf{K})=\sum_{\kappa} a_{\kappa} \sum_{l} X_{l}^{(\kappa)} \mathbf{K}_{l}^{(\kappa)^{*}}$.

The $\mathbf{K}_{l}^{(\kappa) *}$ are formed of products and powers of the components of $\mathbf{K}$ up to the desired order. Both $\mathbf{K}_{l}^{(\kappa)^{*}}$ and $X_{l}^{(\kappa)}$ are constructed with group theoretical methods (see refs. [2-4]). The parameters $a_{\kappa}$ are the specific material constants (e.g. mass parameters, $g$ values and deformation potentials). Table 1 shows the expansion the TRS program is based upon.

The expansion of the Hamiltonian matrix $\mathscr{H}(\mathbf{K})$ allows its separation into parts of different symmetry (compare scheme of Hensel and Suzuki [2]). To this end we change to a new set of canonical variables [3] and choose the direction of magnetic field and stress to be parallel to the [001] direction. Then the eigenstates are expanded into the elements of the orthonormal basis $\left\{\left|\zeta ; n_{a} \alpha M\right\rangle\right\}$ for fixed $\zeta$,

$|\psi(\zeta)\rangle=\sum_{n_{a} \alpha M} C\left(\zeta ; n_{a}, \alpha, M\right)\left|\zeta ; n_{a} \alpha M\right\rangle$,

where

$\zeta$ dimensionless wave vector parallel to magnetic field,

$n_{a}$ Landau oscillator quantum number,

$\alpha$ index of the band $\alpha \in\{c, v, s\}$,

$M$ angular momentum quantum number, $\pm \frac{1}{2}$ in c,s-band, and $\pm \frac{3}{2}, \pm \frac{1}{2}$ in v-band.

Using this basis in the effective-mass Hamiltonian we arrive at a secular problem for the expansion coefficients $C$. TRS solves this secular problem and does not only display the results (energy eigenvalues, eigenvectors) in ordered form, but also uses them to calculate the oscillator strengths of direct dipole transitions.

\section{Description of the program}

\subsection{Organization of the program}

TRS is a program built on a menu structure and therefore intended to run in interactive mode. The combination of a clearly designed menu struc- 
ture and detailed error messages facilitate the operation by the casual user. The menus are ordered as a tree with branches to several levels. Starting from the first level which only contains the main menu the user proceeds by choosing the appropriate items. From level to level the choices become more specific until the program actions themselves are reached which form the leaves of the menu tree. The available choices are displayed as text lines. An item is chosen from a menu by entering the number or character preceding the respective text line. Any data entered in one menu is not affected when switching to and working in another menu. The items of a menu may be selected in any order regardless of previous choices. However, not all choices will start a program action if data from other items are needed which have not yet been entered.

In fig. 1 the main menu is shown. The first three choices allow the access to operations concerning initial data which must be provided in order to process any calculations. These data are divided into three groups:

(1) specific material constants defining the semiconductor's properties;

(2) variable parameters which affect the eigenstates (magnetic field, stress, and wave vector - all parallel to the [001] crystal axis);

(3) data to determine direct dipole transitions.

In all cases the following operations can be per-

formed:

- input from the terminal,

- alteration via terminal,

- display on the terminal,

- input from a file,

- output to a file.
This gives complete control over the input data and enables the user to create a data base of files for his specific materials and applications.

The fourth choice leads to a menu where the calculations are specified and started while the last choice is used to terminate the program. The results of the calculation for all combinations of variable parameters are written to two types of sequential files. The first type is a listing file which is written formatted including FORTRAN printer control and contains all results in printable and readable conformation. The second type is called graphic file. It is written unformatted and therefore contains all results without loss of accuracy by format conversions. Its data may be used for graphical display or further utilizations by other computer programs. Either one of these file types or both together can be generated with each run. A calculation does not change any values whatsoever entered in any menu. Therefore it is not necessary to start the program again, but the old input data can be directly used and altered for the next calculation.

\subsubsection{Subroutines and functions}

The program TRS is divided into the following units,

1 program,

37 subroutines,

6 functions ( 1 integer, 1 character, 4 logical),

7 block data,

amounting to a total of 51 units performing all actions necessary for the operation of TRS. No external units are used. In table 2 the units are ordered according to their function. The actions of

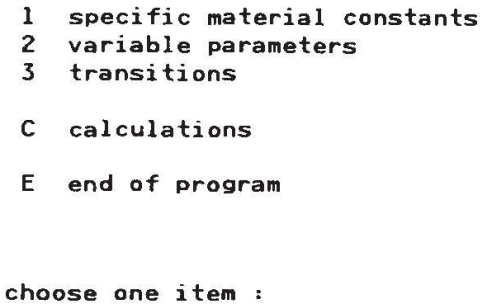


the units and the parameters passed between them are documented in the program listing. Execution begins with unit PROGRAM TRS which contains the start-up, the main menu, and shut-down operations. From this unit the menus of the second level are called (SM21,..,SM24). They control the actions concerning initial data such as display

Table l

Matrix Hamiltonian TRS is based upon

$$
\begin{aligned}
& \mathscr{H}^{\mathrm{wi}}=E_{\mathrm{gap}}+\frac{h^{2} k^{2}}{2 m}(1+2 F)-\left(g_{\mathrm{s}}^{0}-4 N_{1}\right) \frac{e h}{4 m c}(\sigma \cdot H) \\
& +C_{1} \operatorname{tr} \epsilon \\
& \mathscr{H}^{v v}=\mathscr{H}_{k}^{w v}+\mathscr{H}_{k l}^{w v}+\mathscr{H}_{i}^{w v}+\mathscr{H}_{\varepsilon k}^{v v} \\
& \mathscr{H}_{k}^{w}=-\frac{h^{2}}{m}\left(\frac{1}{2} \gamma_{1} k^{2}-\gamma_{2}\left[\left(J_{x}^{2}-\frac{1}{3} J^{2}\right) k_{x}^{2}+\text { c.p. }\right]\right. \\
& \left.-2 \gamma_{3}\left[\left\{J_{x} J_{y}\right\}\left\{k_{x} k_{y}\right\}+\text { c.p. }\right]\right) \\
& -\frac{e h}{m c}\left(\left(\kappa J_{x}+q J_{x}^{3}\right) H_{x}+\text { c.p. }\right) \\
& \mathscr{H}_{k t}^{\mathrm{vv}}=\frac{2 C}{\sqrt{3}}\left(\left\{\left(J_{v}^{2}-J_{z}^{2}\right) J_{x}\right\} k_{x}+\text { c.p. }\right) \\
& \mathscr{H}_{\epsilon}{ }^{\mathrm{v}}=D_{\mathrm{d}} \operatorname{tr} \epsilon+\frac{2}{3} D_{\mathrm{u}}\left[\left(J_{v}^{2}-\frac{1}{3} J^{2}\right) \epsilon_{\mathrm{rv}}+\text { c.p. }\right]
\end{aligned}
$$

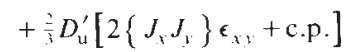

$$
\begin{aligned}
& \mathscr{H}_{\epsilon h}^{\mathrm{wv}}=\left[C_{4}\left(\epsilon_{y y}-\epsilon_{z z}\right) k_{x}+C_{5}^{\prime}\left(\epsilon_{x y} k_{y}-\epsilon_{x z} k_{z}\right)\right] J_{x}+\text { c.p. } \\
& \mathscr{K}^{\mathrm{si}}=-\left(A+\frac{h^{2}}{2 m} \gamma_{1} k^{2}\right)-\kappa \frac{e \hbar}{m c}(\boldsymbol{\sigma} \cdot \boldsymbol{H})+D_{\mathrm{d}} \operatorname{tr} \boldsymbol{\epsilon} \\
& \mathscr{H}^{\mathrm{uv}}=\sqrt{3}\left[P\left(k_{x} T_{x}+\text { c.p. }\right)+\mathrm{i} B\left(T_{x}\left\{k_{y} k_{z}\right\}+\text { c.p. }\right)\right. \\
& \left.+\mathrm{i} C_{2}\left(T_{r} \epsilon_{y z}+\text { c.p. }\right)\right] \\
& +\frac{3 \hbar^{2}}{m} \sqrt{\frac{3}{2}} N_{2}\left[\left(k_{z}^{2}-\frac{1}{3} k^{2}\right)\left(T_{x x}-T_{y y}\right)-\left(k_{x}^{2}-k_{y}^{2}\right) T_{z=}\right] \\
& +\frac{\sqrt{6}}{\mathrm{i}} N_{3} \frac{e h}{m c}\left(H_{x} T_{y z}+\text { c.p. }\right) \\
& \mathscr{H}^{\mathrm{cs}}=-\frac{1}{\sqrt{3}}\left[P^{\prime}(\boldsymbol{k} \cdot \rho)+\mathrm{i} B^{\prime}\left(\rho_{\mathrm{x}}\left\{k_{y} k_{z}\right\}+\text { c.p. }\right)\right. \\
& \left.+\mathrm{i} C_{2}\left(\rho_{\mathrm{c}} \boldsymbol{C}_{\mathrm{yz}}+\text { c.p. }\right)\right] \\
& \mathscr{H}^{\mathrm{vs}}=\mathscr{K}_{k}^{\mathrm{vs}}+\mathscr{H}_{\mathrm{c}}^{\mathrm{vs}}+\mathscr{H}_{\mathrm{ck}}^{\mathrm{vs}} \\
& \mathscr{K}_{k}^{v s}=-\frac{\hbar^{2}}{m}\left[-3 \gamma_{2}\left(U_{x x} k_{x}^{2}+\text { c.p. }\right)-6 \gamma_{3}\left(U_{x, y}\left\{k_{x} k_{y}\right\}+\text { c.p. }\right)\right] \\
& -\frac{e \hbar}{m c} \frac{3}{2} \kappa\left(U_{x} H_{x}+\text { c.p. }\right) \\
& \mathscr{H}_{\mathrm{q}}^{\mathrm{v}}=2 D_{\mathrm{u}}\left(U_{x x^{\prime}} \epsilon_{x x}+\text { c.p. }\right)+2 D_{\mathrm{u}}^{\prime}\left(2 U_{x y} \epsilon_{x y}+\text { c.p. }\right)
\end{aligned}
$$

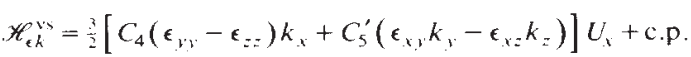

Table 2

List of program units according to their function

\begin{tabular}{ll}
\hline Function & Unit \\
\hline menus & TRS \\
& SM21, SM31, PRTMAT \\
& SM22, IOVP, SVP \\
& SM23, PRTTRA, PRTRAN \\
& SM24, PRTNOS, INPNOS, PRTPPL, \\
& SELPPL \\
menu control & PRTMEN, TITLE, MENLIN. \\
& MENCHO, DECSTK \\
calculation & CALC, BDACD, CDMC, MATRIX, \\
& DIAGON, PRTRES, SITION, \\
I/O & HEADER \\
& CLRSCR. YESNO, HETC, FSWBAR, \\
errors, warnings & TUP \\
assistant & OPNFIL, RDFILN, OPFL \\
& COR, FLDSTT, FLDSTF, TFFES. \\
data & TLCTUC \\
& MENU, MATCON, PARVAR, TRAN, \\
& NOSAPP, WAVFC, PAGE \\
\hline
\end{tabular}

or input managed by further subroutines. All menu operations themselves (generation of menu titles, writing menu lines, request of a choice) are governed by the units comprised as menu control. Main unit for the calculations is subroutine CALC controlling conversion of material constants (BDACD, CDMC), setting up the Hamiltonian (MATRIX), diagonalizing it (DIAGON), printing the results (PRTRES), and evaluating transitions (SITION). Further subroutines for $\mathrm{l} / \mathrm{O}$, error handling, and some assistant functions complete the program.

\subsubsection{COMMON blocks}

Some data are passed between the units by arguments in the respective calls. To reduce the amount of memory needed many subroutines share variables and arrays in certain COMMON blocks. Two groups of these blocks can be distinguished: - calculation data,

- initial and basic data.

The calculation data are shared between the subroutines of the calculation group (see previous section 3.1.1) including dimensionless constants, Hamiltonian matrices, eigenvalues and eigenvectors. Initial and basic data are comprised in the 
seven BLOCK DATA units according to their function.

MENU: definition of complete menu structure, menu lines, choices.

MATCON: initialization of material constants.

PARVAR: initialization of variable parameters.

TRAN: initialization of transition data.

NOSAPP: default values of numbers of states and parts printed in listing file.

WAVFCT: definition of interacting wave functions in [001] direction.

PAGE: definition of printer paper size.

These data are available in all subroutines needed by using COMMON blocks. Since it is clearly laid out the user or programmer may adapt TRS to his own applications very easily. A more detailed description is given in the program listing.

\subsection{Input data}

Four groups of input data are distinguished:

(1) general terminal input and menu handling,

(2) file specifications,

(3) initial data (material constants, variable parameters, transition data),

(4) fixing the scope of calculations.

For the general terminal input, the menu handling, and for file specifications several rules apply which are valid throughout the program: TRS processes both lower case and upper case inputs. Leading blanks of any input are ignored. To avoid

Table 3

List of material constants including test values for $\mathrm{InSb}$

\begin{tabular}{ll}
\hline Cubic elastic compliance constants \\
s11 & {$[1 / \mathrm{Mbar}]$} \\
$\mathrm{s} 12$ & {$[1 / \mathrm{Mbar}]$} \\
$\mathrm{s} 44$ & {$[1 / \mathrm{Mbar}]$}
\end{tabular}

Stress dependent potentials

$\begin{array}{ll}\text { Du } & \mathrm{v} \text {-band splitting 001-strain } \\ \text { Du' } & \mathrm{v} \text {-band splitting } 111 \text {-strain } \\ \text { C4 } & \mathrm{v} \text {-band strain-ind. } k \text {-lin. } 001 \\ \text { C5 } & \mathrm{v} \text {-band strain-ind. } k \text {-lin. } 111 \\ \text { C2 } & \text { cv strain interaction } \\ \text { C1-Dd } & \text { change of gap under strain }\end{array}$

Luttinger parameters

$\begin{array}{ll}\text { gamma 1 } & {[-]} \\ \text { gamma2 } & {[-]} \\ \text { gamma3 } & {[-]} \\ \text { kappa } & {[-]} \\ \text { q } & {[-]}\end{array}$

Further band and gap parameters

\begin{tabular}{|c|c|}
\hline $1+2 F$ & \\
\hline$q-4 N 1$ & eff $a_{-}$actor in $c_{-}$band \\
\hline$c^{6}-4 \mathrm{n} 1$ & eft. $g$-factor in c-band \\
\hline gan & hand gan at $k=0$ \\
\hline Lambda & spin-orbit splitting at $k=0$ \\
\hline Band int & ters \\
\hline $\mathbf{P}$ & kp interaction (cv) lin. \\
\hline B & $\mathrm{kp}$ interaction (cv) quadratic I \\
\hline$P^{\prime}$ & kp interaction (cs) lin. \\
\hline $\mathbf{B}^{\prime}$ & kp interaction (cs) quadratic \\
\hline N2 & $\mathrm{kp}$ interaction (cv) quadratic II \\
\hline N3 & magnetic interaction (cv) \\
\hline
\end{tabular}

$\begin{array}{ll}{[\mathrm{eV}]} & D_{\mathrm{u}}=2.9 \\ {[\mathrm{eV}]} & D_{\mathrm{u}}^{\prime}=4.1 \\ {\left[10^{-7} \mathrm{eV} \mathrm{cm}\right]} & C_{4}=1.7 \\ {\left[10^{-7} \mathrm{eV} \mathrm{cm}\right]} & C_{5}^{\prime}=15.5 \\ {[\mathrm{eV}]} & C_{2}=3.0 \\ {[\mathrm{eV}]} & C_{1}-D_{\mathrm{d}}=-7.0\end{array}$

$s_{11}=2.291$

$s_{12}=-0.764$

$s_{44}=3.180$

$\gamma_{1}^{\mathrm{L}}=40.1$

$\gamma_{2}^{L}=18.1$

$\gamma_{3}^{\mathrm{L}}=19.2$

$\kappa^{\mathrm{L}}=17.0$

$q=0.39$

$\begin{array}{ll}{[-]} & 1+2 F=-5.800 \\ {[-]} & g-4 N_{1}=-3.400 \\ {\left[10^{-11} \mathrm{eV} \mathrm{cm}\right]} & C=9.320 \\ {[\mathrm{eV}]} & E_{\text {gap }}=0.235 \\ {[\mathrm{eV}]} & \Lambda=0.803\end{array}$

$\left[10^{-8} \mathrm{eV} \mathrm{cm]} \quad P=9.973\right.$

[-] B $=3.300$

$\left[10^{-8} \mathrm{eV} \mathrm{cm}\right] \quad P^{\prime}=9.973$

[-] $\quad B^{\prime}=3.300$

[-] $\quad N_{2}=0.230$

[-] $\quad N_{3}=0.230$ 
errors it is recommended not to use the tabulator key. An empty input is created either by just pressing the "〈Enter $\rangle$ " or the equivalent " $\langle\mathrm{Re}$ turn $\rangle$ " key or by entering a string consisting solely of blank characters. In a list of items an empty input has the effect of skipping to the next item without altering the current one. If a file name is requested, an empty input will lead to no file operation at all, i.e. it is assumed that the user does not want to process any file. In all other cases an empty input is ignored. The input of the character "X" (meaning "exit") at any place where an input is requested leads back to the previous menu without any other action. The only exception is the input of a file name: a file may have the name " $X$ ". Any file specification valid on the computer system may be used. If a value is requested the input of the character " $\mathrm{P}$ " followed by a value will purge this value. This is valid only in some cases where this function can be adequately used.

The initial data define the semiconductor's properties and set several variables for the calculations. Table 3 lists the material constants as they show up in the program together with their physical designation used in table 1 and their values set for the test run (compare ref. [3,4]). They are comprised in five groups,

- cubic elastic compliance constants,

- stress dependent potentials,

- Luttinger parameters,

- further band and gap parameters,

band interaction parameters,

which describe the semiconductor including its elastic properties, band gaps, stress induced energy shifts, mixing, etc. The electronic states depend strongly on three different variable parameters:

- wave vector $\left[10^{6} \mathrm{~cm}^{-1}\right]$,

stress [kbar],

- magnetic field [kG].

All calculations are based on a geometry where these parameters are parallel to the [001] crystal axis. Up to 30 different values can be entered for each type of variable parameter. Magnetic fields must be greater than zero while negative or positive values are valid for stresses or wave vectors. This restriction is indispensable because all matrix elements are evaluated in magnetic units. Calculations are performed for all combinations of values of the three types. Variable parameters and material constants are necessary to determine Landau levels. For the evaluation of transitions between them additional boundary values must be entered. These are

- minimum energy of transitions [meV],

- maximum energy of transitions [meV],

- minimum oscillator strength considered, type of transitions determining polarization.

The type of transition is chosen by entering the group representation of initial and final states. TRS uses two such representations. A transition between different representations is circularly polarized, between identical ones it is linearly polarized. All these initial data can be entered from the terminal. Once a complete set was created in the past it is also possible to read these data from previously created files.

After all initial data have been provided the scope of calculations is fixed in the next step. Defaults are preset in BLOCK DATA NOSAPP and are easily changed by the user in advance of compilation. On one hand these are specifications of the eigenstates wanted

- number of Pidgeon-Brown blocks (Landau oscillators),

- number of expansion coefficients printed per eigenvector.

- eigenvalues printed,

- initial and final states of transitions.

On the other hand the user can select the following parts to be included in the listing file

- material constants.

- variable parameters,

- dimensionless constants (magnetic units),

- eigenvalues and eigenvectors,

- transitions,

- matrix Hamiltonian.

Calculations are executed accordingly.

\subsection{Output data}

The output data fall into two groups: output to the terminal and to the files. The terminal output was designed for easy communication between 


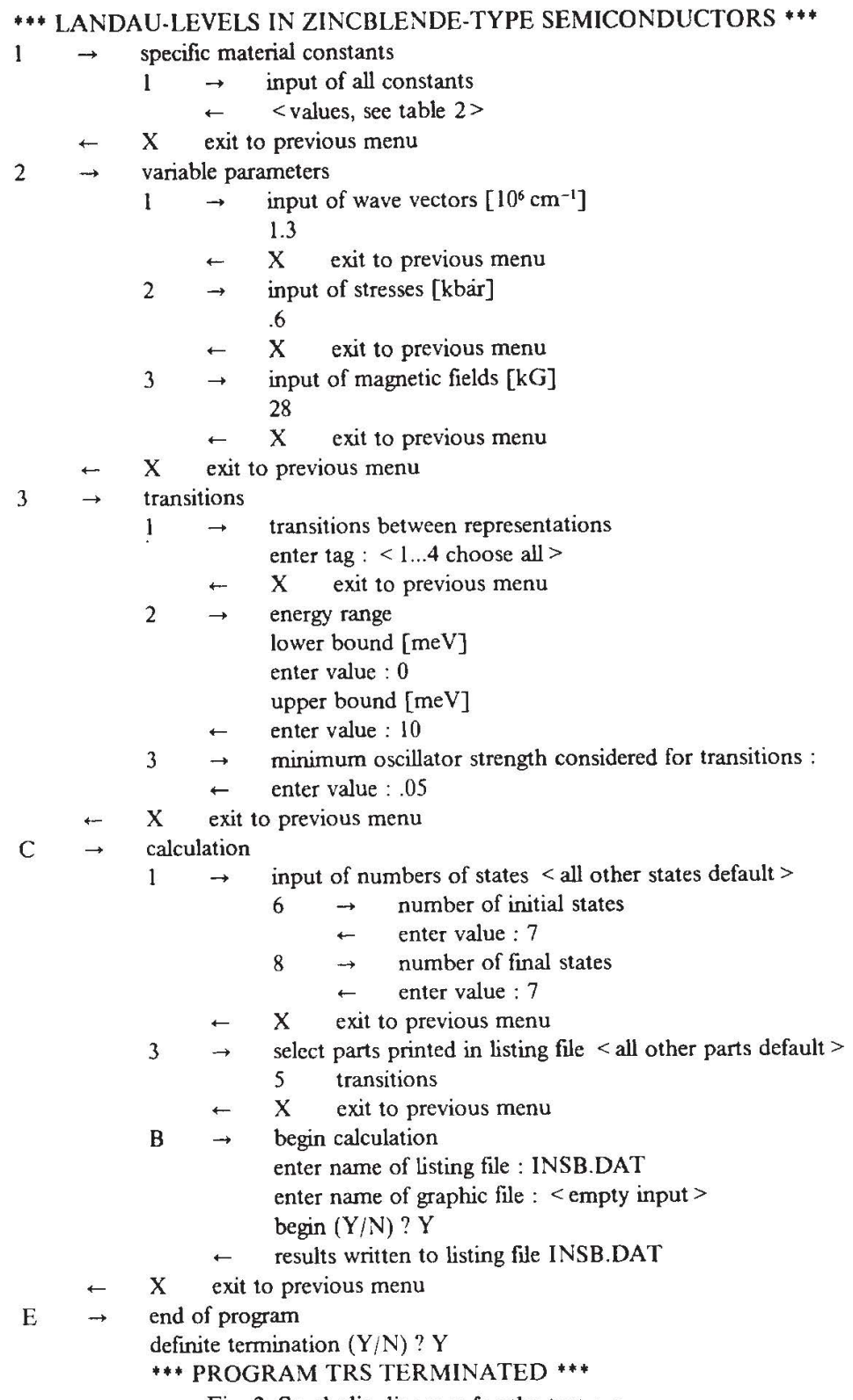

Fig. 2. Symbolic diagram for the test run.

program and user. Its main part are the menus where much effort was exerted to make the menu structure and the corresponding program functions self-explanatory. Furthermore, a large number of error messages and warnings was added which facilitate the program operation and its understanding by the user. They are comprised in three groups.

- 10 FORTRAN I/O errors: the device dependent error number is shown followed by a line specifying the FORTRAN command. Under nor- 
mal conditions no such error should arise. Therefore they are considered fatal and TRS stops.

- 21 warnings and nonfatal run time errors: they are mostly concerned with invalid inputs by the user. Some are simple warnings, others indicate more severe errors, but none of them is fatal. Therefore program execution continues afterwards. If an error condition is met no other operation is performed but the display of the appropriate message. For that reason old data remain intact in most cases.

- 16 errors for debugging assistance: in TRS several safety checks are built in. They are designed as assistance for the programmer during debugging after program changes. Not all possible sources of error are covered but the most important ones are included. All these are fatal and terminate the program. However, none of them should occur under normal conditions.

File output has two functions. First it is used to create a data base of initial data for further calculations. Therefore each group (material constants, variable parameters, transition data) can be both written to files and read again from them. All data are treated unformatted. Therefore no loss of accuracy by FORMAT conversions can occur. Second the output files contain the results of calculations. The listing file is created according to the prerequisites of the numbers of states and the parts to be printed. It is written formatted including FORTRAN printer control and contains the results of the calculations in printable and readable conformation (some pages from the test run are shown at the end of this paper). Creating a graphic file presumes the calculation of both eigenvalues and transitions according to the previously defined numbers of states. It is written unformatted and therefore contains all data without loss of accuracy. The data may be used for graphical display or further utilization by other computer programs. A detailed description of its contents is given in the "TRS User's and Programmer's Guide".

\subsection{Test run}

The test run is performed for the semiconductor InSb. It includes calculations of Landau levels and transitions for one value of wave vector, magnetic field, and stress each. For the number of states and parts printed in the listing file default values from BLOCK DATA NOSAPP are used which are shown in the listing of the test run at the end of this paper. Initial and final states for transitions are chosen from the conduction band. In fig. 2 the operation of the program is illustrated by a symbolic diagram to make clear which menus and items are selected.

\subsection{Device dependence}

Owing to the menu structure of TRS each new page of text should be displayed on a blank screen. Therefore we need a special clear-screen subroutine CLRSCR which naturally is highly device dependent. Consequently, this subroutine must be adapted to the terminal of the computer system. In general we can proceed by sending some necessary control characters to the terminal using simple FORTRAN WRITE statements. On many computers the user may also find a special FORTRAN callable subroutine. This system call should be incorporated in CLRSCR which is easier than to change all calls within the program.

The form of the listing file is automatically adapted to the paper size of the printer. Several essential parameters are comprised in BLOCK DATA PAGE. These are line length and page size. The line length is set with a logical variable LINLEN defining paper widths of 80 or 132 characters per line. The page size is fixed with the integer variable PAGLEN which has a default value of 60 lines per page. The output to the screen is not adaptable. A size of 80 characters per line and 22 lines on the screen is assumed.

\section{References}

[1] E.O. Kane, in: Semiconductors and Semimetals, vol. 1, eds. R.K. Willardson and A.C. Beer (Academic. New York, 1966), p. 75.

[2] K. Suzuki and J.C. Hensel, Phys. Rev. B 9 (1974) 4184.

[3] H.-R. Trebin, U. Rössler and R. Ranvaud, Phys, Rev. B 20 (1979) 686

[4] H.-R. Trebin. W. Wolfstädter, H. Pascher and H. Häfele, Phys. Rev. B 37 (1988) 10249.

[5] G.L. Bir and G.E. Pikus, Symmetry and Strain-Induced Effects in Semiconductors (Wiley, New York, 1974). 


\section{TEST RUN OUTPUT}

*** LANDAU-LEVELS IN ZINCBLENDE-TYPE SEMICONDUCTORS ***

INSB DAT

cubic elastic compliance constants

$\begin{array}{rrrr}1 & 511 & \text { [1/Mbar ] } & 2.29100 \\ 2 & 512 & \text { [1/Mbar ] } & -0.76400 \\ 3 & 544 & \text { [1/Mbar] } & 3.18000\end{array}$

stress dependent potentials

1 Du $\quad v$-band splitting 001-strain [ev] 2.90000

2 Du" v-band splitting 211-strain [eV] 4.10000

3 C4 v-band strain-ind.k-1in. $001[10--7 \mathrm{ev} \mathrm{cm}] \quad 1.70000$

4 C5 v-band strain-ind.k-1 in. $111[10--7 \mathrm{eV} \mathrm{cm}\} \quad 15.50000$

5 C2 cv strain interaction $\quad$ ch 3.00000

6 Cl-Dd change of gap under strain [eV] -7.00000

Luttinger parameters

$\begin{array}{lllr}1 & \text { gammal } & {[-]} & 40.09999 \\ 2 & \text { gamma2 } & {[-]} & 18.09999 \\ 3 & \text { gamma3 } & {[-]} & 19.20000 \\ 4 & \text { Kappa } & {[-]} & 17.00000 \\ 5 & \text { q } & {[-]} & 0.39000\end{array}$

further band and gap parameters

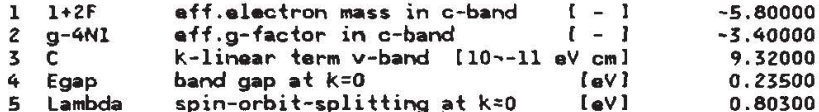

band interaction parameters

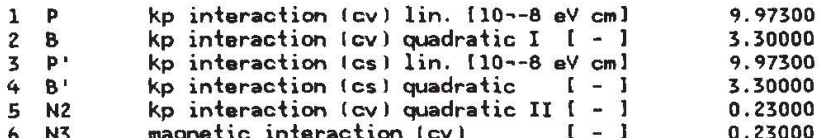

*** LANDAU-LEVELS IN ZINCBLENDE-TYPE SEMICONDUCTORS ***

INSB DAT

number of blocks 24

expansion coefficients 4
eigenvalues printed

wave vector $[10-6 / \mathrm{cm}]: \quad 1.3000$

$\begin{array}{rr}\text { stress } & 0.6000 \\ \text { magnetic field [Kbar] }[\mathrm{KG}]: & 28.0000\end{array}$

REPRESENTATION I - eigenvalues and eigenvectors from 30V $15 \mathrm{C}$ 15S functions

Eigenvalues

$\begin{array}{llllllll}434.4192 & 427.6353 & 416.7446 & 409.7966 & 397.8677 & 390.6125 & 377.5063 & 369.7544\end{array}$ 


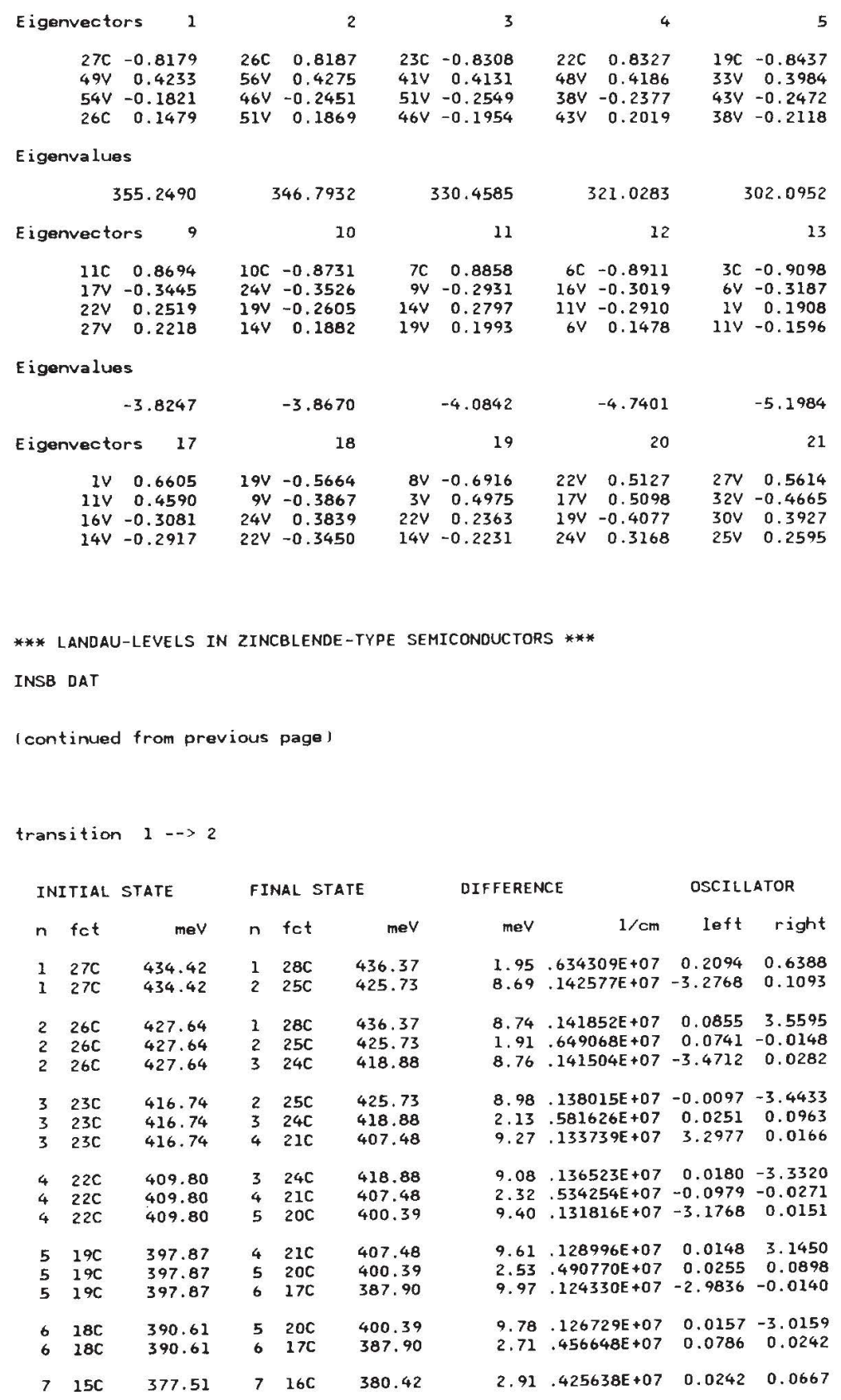

